MATEC Web of Conferences 19, 01005 (2014)

DOI: $10.1051 /$ matecconf/ 20141901005

(C) Owned by the authors, published by EDP Sciences, 2014

\title{
MIXED CONVECTION IN TECHNOLOGICAL RESERVOIR OF THERMAL POWER STATION
}

\author{
Geniy V.Kuznetsov, Vyacheslav I. Maksimov, Aleksander S. Krasnoshlykov \\ Institute of Power Engineering, National research Tomsk Polytechnic University, Tomsk 634050, \\ Russia
}

\begin{abstract}
The problem of mixed convection of a viscous incompressible fluid in an open rectangular reservoir with inlet and outlet of mass with considering nonuniform heat sink at the external borders of the solution domain is solved. The region of the solution was limited by two vertical and by one horizontal walls of finite thickness and one free surface. The flat nonstationary mixed convection within the framework of Navier-Stokes model is examined for liquid and thermal conductivity for solid walls. Distributions of hydrodynamic parameters and temperatures with different intensity of heat sink on the outer contour of the cavity show a change in the intensity of heat sink on the region boundaries of the solution leads to scale changes in the structure of flow and temperature fields of the liquids.
\end{abstract}

\section{Introduction}

The scales of modern power engineering are such, that its interaction with the environment has not only local, but also global in nature [1,2]. When assessing the impact of power engineering on nature, finding measures for the limitation of the negative consequences of this action, and also for creating the optimum thermal systems for environmental protection is necessary the analysis of different hydrodynamic processes. It is essential to the hydrothermal regime of the reservoir- coolers with hot water from the turbine condensers [1,2]. This forecast is important both from the point of view of the thermal pollution of aqueous medium and from the point of view of the temperature distribution of water recirculation. When designing such reservoirs and selection of their technological parameters the need for analysis of thermal conditions of objects, which are the cavity, filled with an incompressible fluid and the sources of inlet and outlet of mass, significant temperaturegradients and heat exchange on the outer contour of cavity. Analysis of the physical picture of flow shows that under such conditions must be realized the regime of mixed convection, complicated by heat sink from the outer boundaries of the analysis region. In many in practice significant cases this heat sink plays important role in the formation of the thermal condition of object and water flows. Up to now the simulation of such flows in the combined setting, which considers the influence of environment on the flow pattern and the temperature field of object was not carried out [1-5]. The purpose of this work is the numerical simulation of the mixed convection of liquid in the open reservoir with the local sources of input and output of mass considering the heat sink to the environment around the outer contour.

This is an Open Access article distributed under the terms of the Creative Commons Attribution License 4.0, which permits unrestricted use, distribution, and reproduction in any medium, provided the original work is properly cited. 


\section{Physical model}

Is examined the flow of the incompressible viscous fluid and heat exchange into the cavity of that having two vertical, one horizontal walls of finite thickness and one free surface, with two sections of input and output of liquid (Fig. 1).

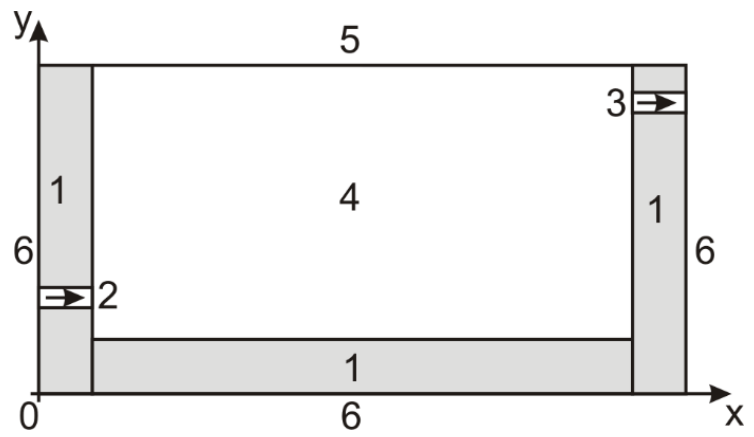

Figure 1. Area solutions: 1) solidphase; 2, 3) sections of input and output of liquid into the cavity; 4) liquid; 5) free surface; 6) external borders.

When the problem is assumed that the temperature of the injected fluid substantially exceeds the initial temperature of the medium in the cavity and is considered known. Mass arrival is also known value. Heterogeneous boundary conditions are assigned on the outer boundaries of region. Heterogeneity is caused by different thermophysical characteristics of media and by conditions of heat exchange on the boundaries of the solution domain. Liquid is considered viscous, heat-conducting, laminar flow regime. Mass outflow from the upper limit due to evaporation is not considered. All boundaries except the sections of inlet and outlet of mass are considered liquid-tight. The thermophysical properties of liquid and solid phase do not depend on temperature.

Laminar flow regime is also most typical for the attainable in the task in question values ofthe Reynolds and Grashofnumbers. The draining of mass from the free surface of reservoir due to the evaporations is, as a rule, achieved in practice, but the intensity of this process is not so great so that it could change the configuration of cavity by the filled water, or its sizes.

Task is examined in the nonstationary setting due to the ability of the material of solid walls to accumulate by heat- and significant temperature differentials between the introduced into the cavity liquid and the initial temperature of solid walls. In the general case different arrangements of openings for input and output of liquid from the cavity are possible. Are examined the most typical versions (Fig. 1), realization of which, leads to the formation of the structures of flow of liquid most interesting for the analysis. It is also assumed with the formulation of the problem that the contact on the interfaces "liquid - solid wall" is ideal, but heat exchange conditions on the outer boundaries of the solution region do not depend on time. The ideality of the contact of liquid and solid wall in the task in question is much more substantiated, than for example in the analogous models for the contact of two solids body. Liquid fills all roughness elements of concrete (or soil) in the reality. Therefore heat transfer through this contact surface in practice insignificantly is differed from ideal. Also in the real practice the characteristic times of a change of heat exchange conditions on the free surface of technological reservoirs are much more than the characteristic transit time of energy in the liquid both due to the convection and due to the thermal conductivity. Special importance in in this problem are the boundary conditions on the outer boundaries of the solutionregion (Fig. 1). These conditions determine the intensity of heat sink from the outer boundaries and essential influence on the flow conditions of liquid, and, correspondingly, to the intensity of heat transfer. . Based on the analysis of the various embodiments of the scheme in practice can be on the right, left and bottom borders of the region solutions exhibit boundary conditions of various kind. For the correct use of fourth kindconditions necessary to enlarge the dimensions of the solution region by the introduction of additional subregions to the values $\mathrm{x}$ and $\mathrm{y}$, with which the front of warming up in any regimes does 
not reach these outer boundaries. This approach substantially complicates the algorithm of problem solution. The use of heat insulation conditions on the outer boundaries of the solution region (ris.1) is not legitimate, since under the actual conditions with different gradients of temperatures the heat sink into the environment from these boundaries is always accomplished. The boundary conditions of the second kind on the discussed boundaries were selected for these reasons.

It should be noted that on the boundaries of the enclosing walls the use of boundary conditions of the second kind during the entire apparent formalization of real physical process can be substantiated sufficiently reasoned. Thus, for instance, is possible solution of the problem for the relatively small time interval in question, but taking into account heat propagation directly in the ground. Boundary conditions ofsolutioncan define a range of possible changes in the heat flux on the border "concretesoil". This characteristic will change in the time very slowly in connection with the high energy content of real soil. Accordingly, this solution creates objective conditions for using the boundary conditions of the second kind on the outer duct of reservoir.

\section{Mathematical model and method of solution}

Transfer process of mass, momentum and energy in such a setting is described by the unsteady Navier-Stokes equations for the liquid phase and the heat equation for the solid phase [6-8]. The problem was solved in the dimensionless formulation.

Accordingly, the dimensionless Navier-Stokes equations in the Boussinesq approximation in variables "vorticity - stream function - temperature" for the liquid phase (mixed convection mode) and the heat equation for the solid phase will be:

$$
\begin{gathered}
\frac{\partial \Omega}{\partial \tau}+U \frac{\partial \Omega}{\partial X}+V \frac{\partial \Omega}{\partial Y}=\frac{1}{\operatorname{Re}}\left(\frac{\partial^{2} \Omega}{\partial X^{2}}+\frac{\partial^{2} \Omega}{\partial Y^{2}}\right)+\frac{\mathrm{Gr}}{\operatorname{Re}^{2}}\left(\frac{\partial \Theta}{\partial X}\right), \\
\frac{\partial \Theta}{\partial \tau}+U \frac{\partial \Theta}{\partial X}+V \frac{\partial \Theta}{\partial Y}=\frac{1}{\operatorname{Re} \cdot \operatorname{Pr}}\left(\frac{\partial^{2} \Theta}{\partial X^{2}}+\frac{\partial^{2} \Theta}{\partial Y^{2}}\right), \\
\frac{\partial^{2} \Psi}{\partial X^{2}}+\frac{\partial^{2} \Psi}{\partial Y^{2}}=\Omega, \\
\frac{1}{\text { Fo }} \frac{\partial \Theta}{\partial \tau}=\frac{\partial^{2} \Theta}{\partial X^{2}}+\frac{\partial^{2} \Theta}{\partial Y^{2}} .
\end{gathered}
$$

Here $\mathrm{Gr}=\mathrm{g} \beta L^{3} \Delta T / v^{2}-$ Grashof number; $\beta$ - Thermal coefficient of volume expansion; $g-$ acceleration created by the mass forces; $\operatorname{Re}=2 V L / v-$ Reynolds number; $\operatorname{Pr}=v / a-$ Prandtl number; Fo $=a t_{0} / L^{2}-$ Fourier number; $a$ - coefficient of thermal diffusivity; $v-$ the kinematic viscosity coefficient.

Initial conditions:

$$
\Psi(X, Y, 0)=0, \Omega(X, Y, 0)=0, \quad \Theta(X, Y, 0)=0 .
$$

Boundary conditions:

- on the outer contour of the area under consideration (except the free liquid surface) are given boundary conditions of the second kind

$$
\frac{\partial \Theta}{\partial n}=\mathrm{Ki}
$$

- on the free surface of the liquid

$$
\frac{\partial^{2} \Psi}{\partial Y^{2}}=\tau_{z}, \frac{\partial \Theta_{f}(X, Y)}{\partial Y}=\mathrm{Bi} \cdot \Theta_{f}(X, Y)+\mathrm{Bi} \cdot \frac{T_{0}-T_{e}}{T_{i n}-T_{0}}
$$

- internal border between the solid and liquid phases, parallel to the axis $0 \mathrm{Y}$ : 


$$
\Psi=0, \frac{\partial \Psi}{\partial Y}=0, \Theta_{w}=\Theta_{f}, \quad \frac{\partial \Theta_{w}}{\partial Y}=\lambda_{w, f} \frac{\partial \Theta_{f}}{\partial Y} ;
$$

- internal border between the solid and liquid phases, parallel to the axis $0 \mathrm{X}$

$$
\Psi=0, \frac{\partial \Psi}{\partial X}=0, \Theta_{w}=\Theta_{f}, \quad \frac{\partial \Theta_{w}}{\partial X}=\lambda_{w, f} \frac{\partial \Theta_{f}}{\partial X} ;
$$

- section of inlet liquid

$$
\Theta=1, \frac{\partial \Psi}{\partial Y}=1
$$

- section of outlet liquid

$$
\frac{\partial \Theta(X, Y)}{\partial Y}=0, \frac{\partial \Psi}{\partial Y}=1 ;
$$

here $X, Y$-dimensionless coordinates corresponding $x, y ; L$ - length of the cavity along the axis $\mathrm{x} ; \tau-$ dimensionless time; $U, V$ - dimensionless speeds; $V_{\text {in }}$ - scale of speed (the velocity of the liquid inlet); $\Theta$ - dimensionless temperature; $T_{0}$ - temperature of liquid and solid at the initial time; $T_{i n}$ - entering fluid temperature (2, fig. 1); $\Psi$ - dimensionless analog stream function; $\Omega$ - dimensionless analog vorticity; $\mathrm{Ki}=q L / \lambda_{w}\left(T_{i n}-T_{0}\right)-$ the number of Kirpicheva; $\tau_{z}=\tau_{z}^{\prime} L / V_{\text {in }} \mu$ - dimensionless shearing stress; $\mathrm{Bi}=\alpha L / \lambda$ - Biot number; $\alpha$ - the coefficient of heat exchange between the environment and the region of the solution by the boundary of $\mathrm{y}=0$ in question; $T_{e}-$ Environment temperature; $\lambda_{w}-$ thermal conductivity coefficient of solid phase; $\lambda_{f}$ - thermal conductivity coefficient of liquid phase; $\lambda_{w, f}$ - the relative thermal conductivity coefficient; $q$ - heat flux on the outer boundaries of the solution region (6, fig. 1$) ; \tau_{z}^{\prime}-$ shearing stress of friction on the free surface $(5$, fig. 1$) ; \mu-$ the dynamic viscosity of liquid.

The system of equations (1) - (4) with the appropriate initial and boundary conditions is solved by finite difference method [6-8].

Equations (1) - (4) were solved sequentially. Each time step starts with the evaluation of the temperature field in the liquid and solid wall elements (equation 2.4), and then solved the Poisson equation for the stream function (3). Further defines the value of the vorticity vector at the boundaries of the cavity, and solve the equation of motion (1).

In solving the system of equations (1-3) as the initial approximation used stationary solutions obtained at low Reynolds numbers.

\section{Analysis of numerical simulation results}

Numerical studies have been conducted for the following values of the dimensionless anddimensional quantities: $\operatorname{Pr}=7,1,100 \leq \mathrm{Re} \leq 1000, \mathrm{Gr} \leq 10^{5}, T_{0}=293 \mathrm{~K}, 300 \leq T_{\text {in }} \leq 343 \mathrm{~K}, 273 \leq T_{e} \leq 293 \mathrm{~K}$.

It should be noted that the ranges of the dimensionless quantities and size characteristicswere selected based on the following conditions. First - matching range of parameters inreal industrial waters.Second - the real scales of existence both of the forced and free convection without the degeneration of these mechanisms of heat transfer. Furthermore, were controlled the conditions of the geometric similarity of real systems and used in the mathematical description of process models. In this case also were examined in essence not the most favorable for the realization "conjugate" heat transfer [8] ranges of change both the dimensional values and dimensionless complexes. Thus, for instance an increase in the temperature of the introduced into the cavity in question liquid by $20 \ldots 30^{\circ}$ could substantially change the relationship between the characteristic times of the realization of the process of the forced and free convection, but extremely possible versions were not examined.

Fig. 2 shows typical results of the solution of (1-4) with varying intensity heat sink on the borders of the outer contour. 

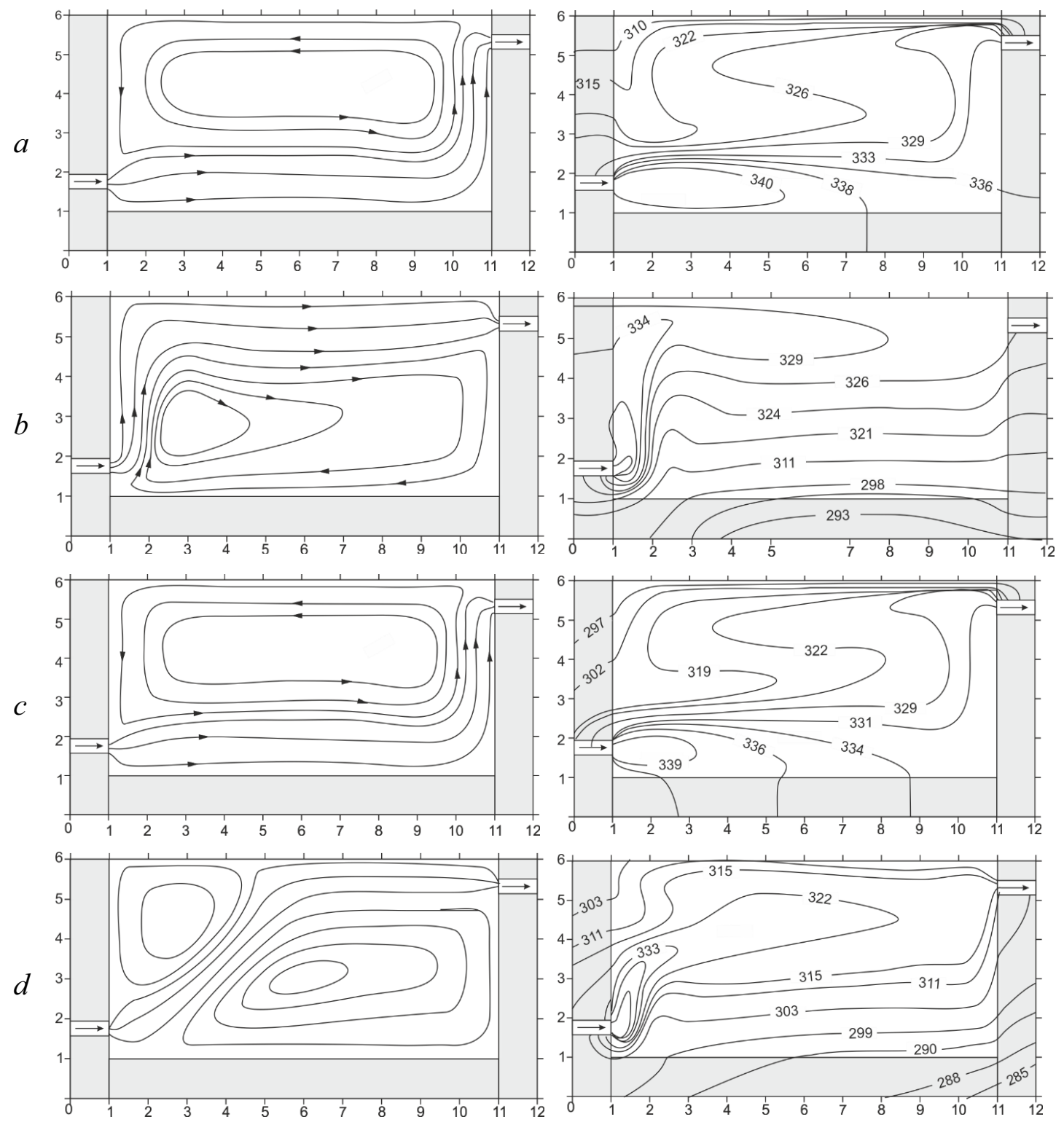

Figure 2. Flow and temperature field for mixed convection model with various heat sink on external borders: a on top, $\mathrm{b}$ - on the bottom, $\mathrm{c}$ - on the left, $\mathrm{d}$ - on the right. $\operatorname{Pr}=7.1, \mathrm{Gr}=10^{5}, \mathrm{Re}=1000$. Dimensions are given in $\mathrm{m}$, temperatures $-\mathrm{K}$

Fig. 2a shows the streamlines and temperature field for the case when the left, right and bottom borders of the outer contour insulated. At the top of the cavity seen one an extensive vortex that is formed by natural and forced convection. In this case the heated liquid due to natural convection seeks upwards. Accordingly, the cold liquid, falling toward the left side, running out the main flow to the bottom of the cavity. Due to the high intensity of the vortex, the heated liquid rises up along the right side only to the outlet. In the cavity is a relatively uniform temperature distribution due to the fact that the liquid is cooled only at the upper boundary and the heat sink on the other boundaries is absent.

Fig. $2 \mathrm{~b}$ shows the streamlines and temperature field for the case when the lower boundary of the outer contour of the heat sink is present, and the left and right boundaries are insulated. It also shows 
the formation of a vortex extensive, but already at the bottom of the cavity. The main mechanism of its formation are forced convection forces. Hot liquid due to the forces of natural convection rises. In the presence of heat removal from the lower boundary of the external contour, cooled liquid moving along the bottom directs the main stream to the left wall and the upper boundary of the cavity. The temperature distribution in this case is substantially uneven. In the upper part of the cavity is almost 50 degrees temperature higher than the bottom. This happens due to the fact that the coolant in the lower part of the cavity is practically not mixed with the main heated stream.

Fig. $2 \mathrm{~d}$ shows the streamlines and temperature field for the case, when on the right boundary of outer contour there is heat sink, and lower and right boundary are heat-insulated. Seen the formation of two vortices in a cavity under consideration. Upper vortex exists due to the forces of the natural and forced convection. Flow relative to cold liquid descends on the left wall, pressing input flow downward. Lower vortex is formed due to the forced convection, as a result the motion of main flow and free convection during cooling of liquid by heat sink from the right boundary of cavity. Vortex in the lower region is scale than the vortex in the upper region, since the size of upper vortex is limited to the being risen flow of hot liquid. Temperature field is sufficiently even, since the main flow is passed practically along the diagonal of cavity. A drop in the temperature not more than 35 degrees, since 2 vortices are formed, in which sufficiently intensively occurs the heat exchange with the main flow.

The distributions of the hydrodynamic parameters and temperatures with different intensity of heat sink on the outer duct of the cavity in question show that, a change in the intensity of heat sink on the boundaries of solution region leads to scale changes in the structure of flow and temperature fields of liquids.

All presented as illustrative materials are obtained for the regimes sufficient moderate mixed convection. Is in practice possible the achievement, for example, of the large scales of temperatures or intensities of heat sink on the external contour. In such cases chosen regularities are manifested substantially more distinctly.

\section{CONCLUSION}

The influence of the varied conditions of heat exchange on the boundaries of rectangular cavity with the sources of inlet and outlet of mass to the regimes of the convection of the viscous incompressible fluid into the conjugate formulation is theoretically investigated. The obtained theoretical consequences give the new information, which characterizes convective flow regime and more for construction and approval of models of mixed convection in the conjugate formulation.

On the basis obtained results it is possible to make the conclusion that by regulating the intensity of heat exchange on the boundaries of rectangular region it is possible to govern the thermal regime of the liquid with implementation mixed convection under the conditions for inlet and outlet of mass into the region of limited sizes.

Work performed under the research state assignment «Science» (Code of Federal Target Scientific and Technical Program 2.1321.2014).

\section{References}

1. T.P. Dotsenko, Reservoirs and Water Barrier hydropower facilities, Energoatomizdat, 1989.

2. TV.M. Popov, V.I. Ryabcev, G.A. Ryabcev, Life Safety, 4 (2002).

3. B.E. Rossi di Schio, E., International Journal of Heat and Fluid Flow 24, 6 (2003)

4. G. Rosengarten, International Journal of Heat and Fluid Flow 22, 2 (2001)

5. S.A. Ivanenko, P.P. Koryavov, Mathematical modeling 14, 6 (2002)

6. G.V. Kuznetsov, V.I. Maksimov, Journal of Engineering Thermophysics 16, 4 (2007)

7. G.V. Kuznetsov, N.I. Kurilenko, V.I. Maksimov, G.Ya. Mamontov, T.A. Nagornova, Journal of Engineering Physics and Thermophysics, 86, 3 (2013)

8. G.V. Kuznetsov, M.A. Sheremet, Thermophysics and Aeromechanics 16, 1 (2009) 